\title{
Mutual Coupling between Parallel Half-Mode Substrate Integrated Waveguides
}

\author{
Q. H. Lai ${ }^{1}$, C. Fumeaux ${ }^{2}$, W. Hong ${ }^{3}$ \\ ${ }^{1}$ East China Research Institute of Electronic Engineering, Hefei, 230088, P. R. China \\ ${ }^{2}$ School of Electrical and Electronic Engineering, The University of Adelaide, Adelaide, South Australia 5005 \\ ${ }^{3}$ State Key Lab. of Millimeter Waves, Southeast University, Nanjing, 210096, P. R. China
}

\begin{abstract}
This paper presents an experimental study on the mutual coupling between pairs of parallel half-mode substrate integrated waveguides (HMSIWs) in three different configurations. The three different arrangements correspond to the cases where, firstly the open sides of the two HMSIWs face each other; secondly they share the linear array of metallic vias at their closed side; and thirdly the open side of one HMSIW faces the closed side of the second one. As a reference, the mutual coupling between pairs of parallel microstrip lines is also measured and compared to corresponding HMSIWs. The investigation shows that the best isolation can be achieved for the third configuration. The presented results and comparisons are of importance to the application of HMSIWs in the design of microwave integrated circuits (MICs) and array antenna feeds.
\end{abstract}

\section{INTRODUCTION}

Half-mode substrate integrated waveguides (HMSIWs) are planar transmission lines that present narrower transverse size and wider mono-mode operation range as compared to a substrate integrated waveguide (SIW). They can also exhibit lower loss than a microstrip line, particularly in the millimeter-wave range [1]. Those advantages make the HMSIW a potential option for high-speed interconnection application. In the perspective of utilizing the HMSIWs in the design of microwave integrated circuits (MICs), it is essential to study the crosstalk between closely spaced HMSIWs. A previous study has shown that, a pair of parallel HMSIWs that shares a common row of metallic vias as their closed sides can exhibit far-end crosstalk nearly $20 \mathrm{~dB}$ lower than a pair of microstrip lines, when the coupled lines are fabricated on a 0.5 mm thick Rogers 5880 substrate for operation in Ka band [2]. Besides this specific example, there is, to the best knowledge of the authors, no further investigation of the mutual coupling between HMSIWs at this time in the available literature. Therefore, this work focuses on quantitatively characterizing the crosstalk of parallel HMSIWs in different configurations on standard substrates, and comparing this crosstalk to the corresponding results obtained with microstrip lines.

\section{CONFIGURATIONS AND PRACTICAL CONSIDERATIONS}

Fig. 1 illustrates photographs of four selected prototypes of coupled lines, including three pairs of coupled HMSIWs and a pair of microstrip lines. Fig. 1(a) shows the first coupling configuration, where the open sides of both parallel HMSIWs face each other. Each HMSIW branch is fed at both ends by standard a $50 \Omega$ microstrip line, through a tapered transition. Fig. 1(b) shows the second arrangement of coupled HMSIWs, where both HMSIW branches share a common row of metallic vias as their respective closed side.
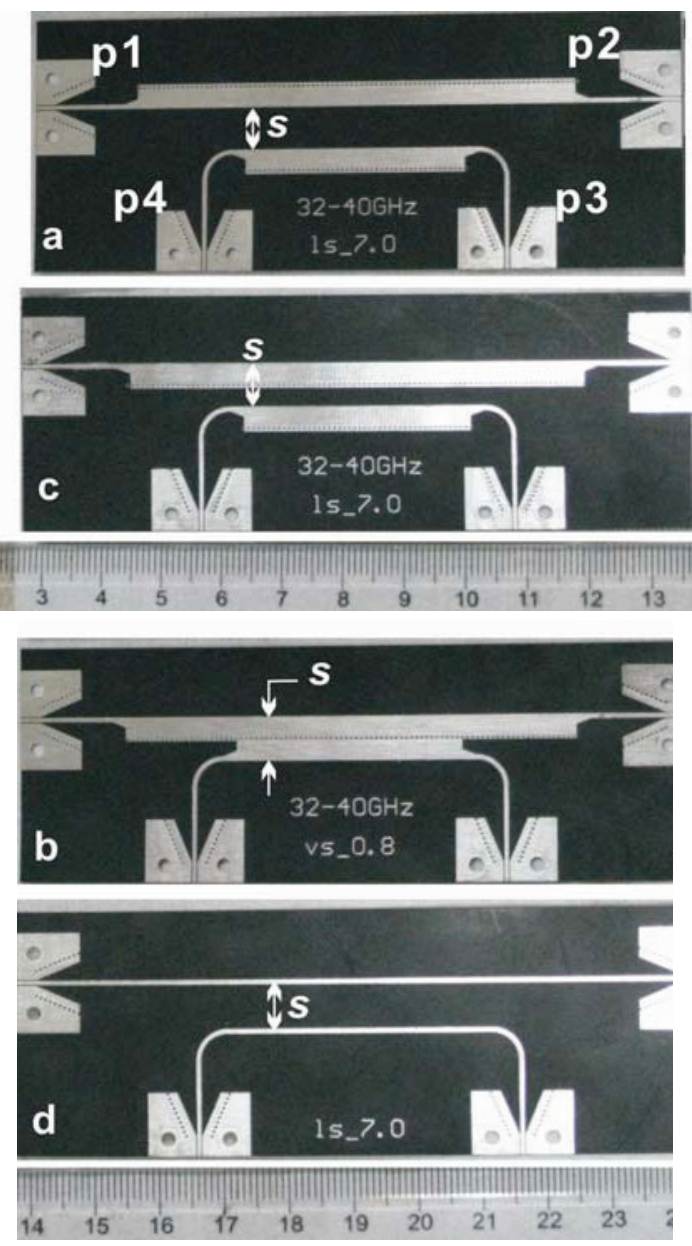

Fig. 1 Photographs of fabricated coupled HMSIWs and microstrip lines: (a) open side to open side, (b) common closed side, (c) open side to closed side, and (d) coupled microstrin lines. 
In the third coupling structure as shown in Fig. 1(c), the open side of one HMSIW faces the closed side of the other. Fig. 1(d) illustrates a pair of coupled standard $50 \Omega$ microstrip lines that is used as a reference in this work. The three HMSIW coupling configurations in Fig. 1 (a-c) are referred in the following as "open side to open side", "common closed side", and "open side to closed side", respectively.

The analysis is conducted in the Ka band (26.5 - 40GHz), therefore the coupled lines are fabricated on a Rogers 5880 substrate with standard thickness of $0.254 \mathrm{~mm}$. This allows a direct comparison with a corresponding microstrip and stands in contrast to the thicker substrate of $0.5 \mathrm{~mm}$ selected in [2]. The width of the HMSIW is $w=3.5 \mathrm{~mm}$. The diameter of metallic vias is $d=0.4 \mathrm{~mm}$ and their interspacing $s_{v}=0.8 \mathrm{~mm}$. Those dimensions results in a cutoff frequency of around 15.0 $\mathrm{GHz}$ for the HMSIWs, which yields an optimal operation range covering the Ka band, as shown in Fig. 2. The spacing $s$ between the parallel HMSIWs is defined as the distance between their open edges, i.e. between the points where the maximum electric field magnitude occurs. For the reference coupled microstrip lines, the spacing $s$ corresponds to the distance between midpoints of the two strips.

Three practical considerations have been taken into account to reduce parasitic effects when designing the test structures for the measurement of the crosstalk between the waveguides. Firstly, in order to reduce the impact of mutual coupling between the tapered transitions, the two parallel HMSIWs are designed with different lengths to avoid parasitic crosstalk from parallel tapered transitions in close proximity. Secondly, for mitigating the direct coupling between ports, the two ports of the shorter HMSIW are placed on the lower side of the substrate. And finally, the Ka band is divided into two subband with separately optimized transistions so as to minimize standing waves.
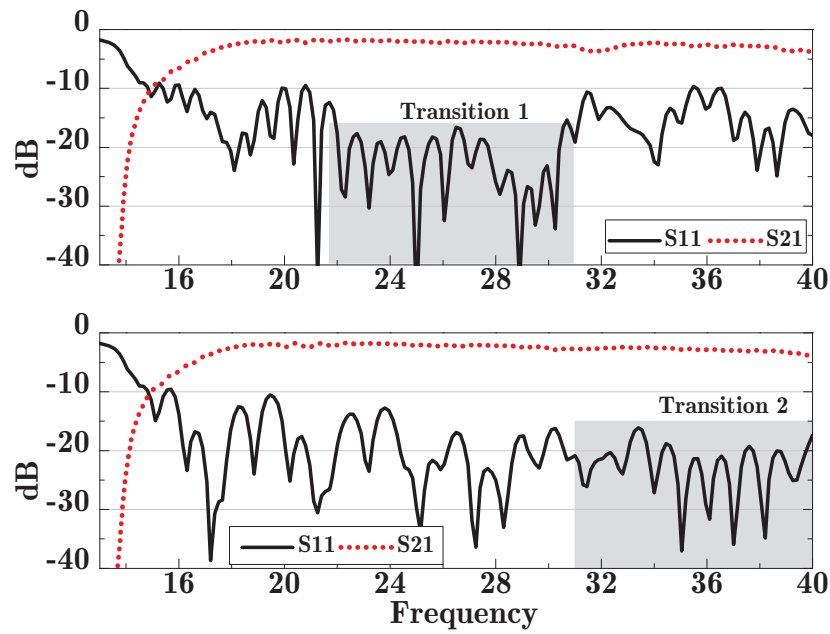

Fig. 2 Measured reflection and transmission coefficients of thru HMSIWs fed by microstrip lines through a tapered transition "1" for $22.5 \mathrm{GHz}-31.0$ $\mathrm{GHz}$ (top) and through a tapered transition "2" for $31.0 \mathrm{GHz}-40.0 \mathrm{GHz}$ (bottom).
Fig. 2 plots the measured reflection and transmission coefficients of a thru HMSIW that is fed by both types of optimized tapered transitions. The results show that a S11 below $-15 \mathrm{~dB}$ is achieved in the frequency range from 22.5 $\mathrm{GHz}$ to $31.0 \mathrm{GHz}$ for Transition 1, and in the range from 31.0 $\mathrm{GHz}$ to $40.0 \mathrm{GHz}$ for Transition 2 .

\section{Mutual Coupling}

The crosstalk between HMSIWs in the "open side to open side" configuration is investigated first. Fig. 3(a) illustrates the measured far-end coupling coefficient S31 obtained at three spacings $s$ equal to $1.0 \mathrm{~mm}, 4.0 \mathrm{~mm}$ and $7.0 \mathrm{~mm}$. The results

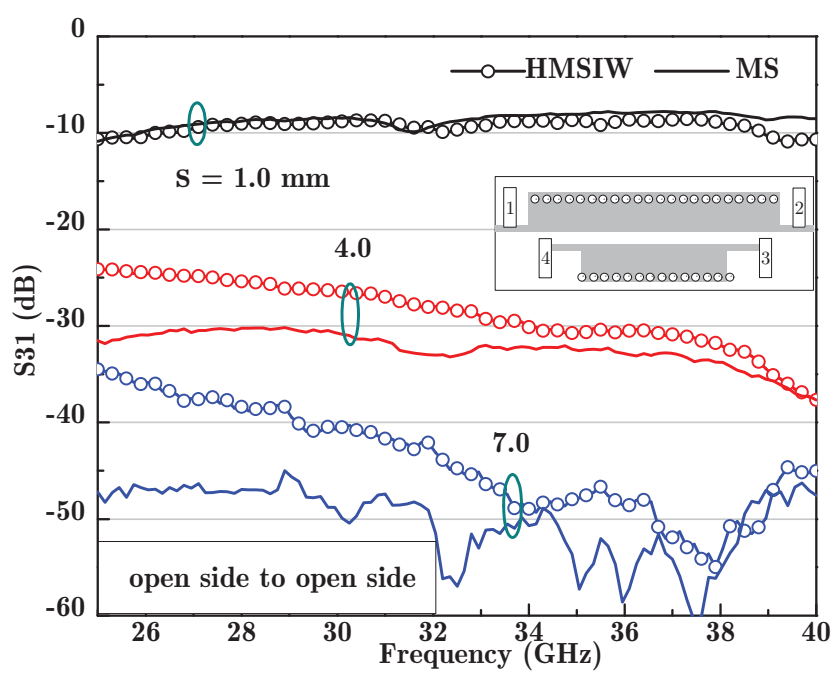

(a)

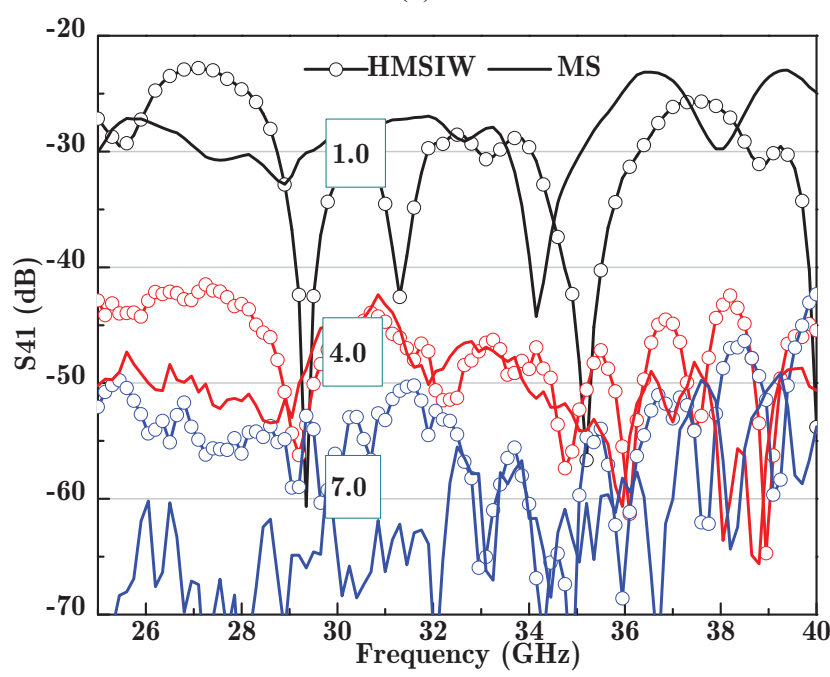

(b)

Fig. 3 Comparison of measured mutual coupling between a pair of parallel HMSIWs vs. a pair of parallel microstrip lines, at different interspacings of $1.0 \mathrm{~mm}, 4.0 \mathrm{~mm}$ and $7.0 \mathrm{~mm}$. The coupled HMSIWs are placed in the configuration of "open side to open side". (a) Far-end coupling, (b) near-end coupling. 


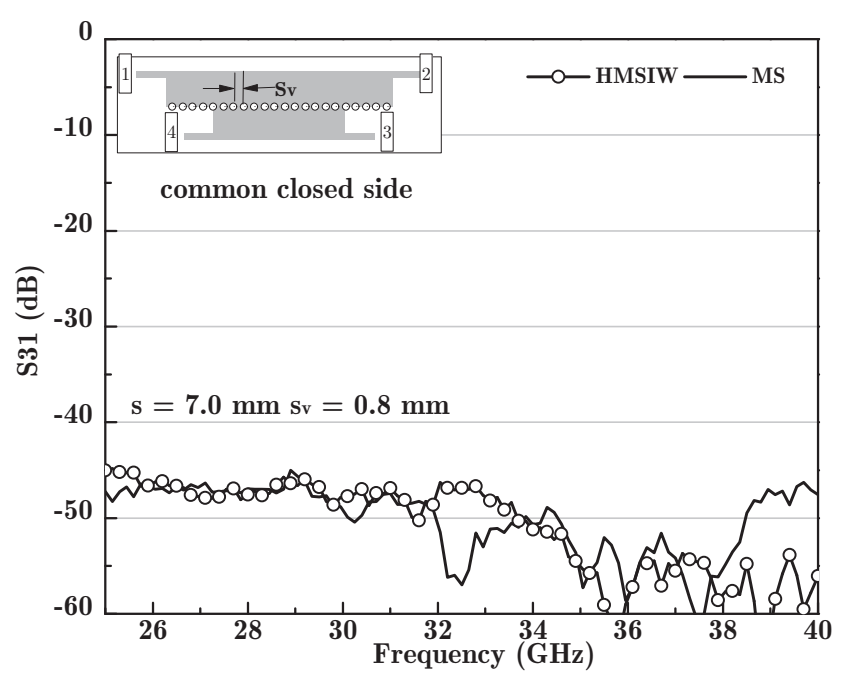

(a)

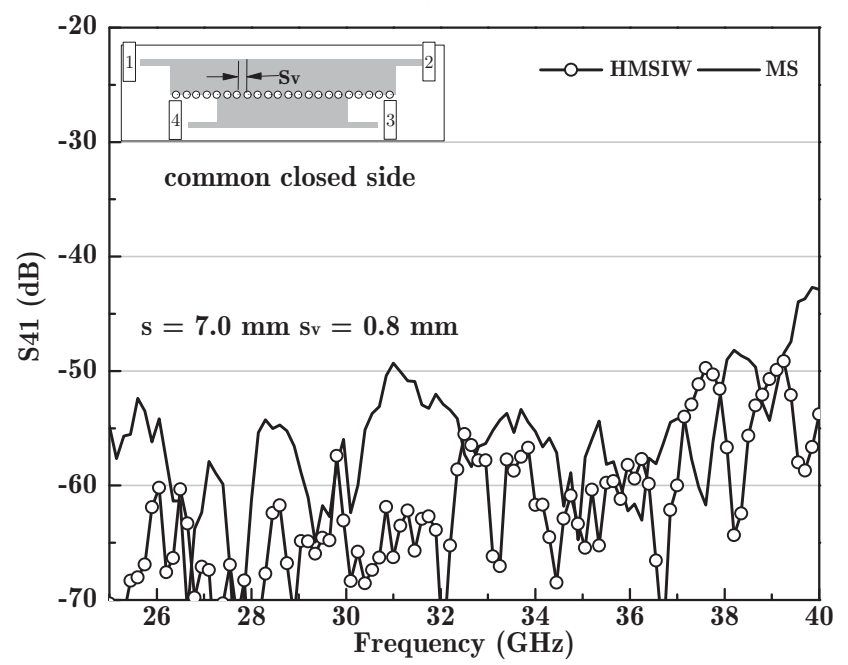

(b)

Fig. 4. Comparison of measured mutual coupling between a pair of parallel HMSIWs and a pair of parallel microstrip lines. The coupled HMSIWs use a llic n closed side. (a) Far-end coupling, (b) near-end coupling.

show that the coupling level for the HMSIWs is close to that for the microstrip lines at a relatively small spacing $s=1.0 \mathrm{~mm}$. With the increasing distance of separation, the mutual coupling level of both the HMSIWs and the microstrip lines significantly decreases. However, the radiation leakage from the HMSIWs in the substrate causes the crosstalk between the HMSIWs to be higher than that between microstrip lines. As the frequency of operation increases, the radiation leakage continuously decreases [3], and this reduces the crosstalk of the HMSIWs above $34 \mathrm{GHz}$ to a level within a few $\mathrm{dBs}$ from that of the coupled microstrip lines. Fig. 3(b) presents the measured near-end crosstalk S41, showing generally similar levels for the HMSIWs and the microstrip lines, regardless of the spacing $s$.

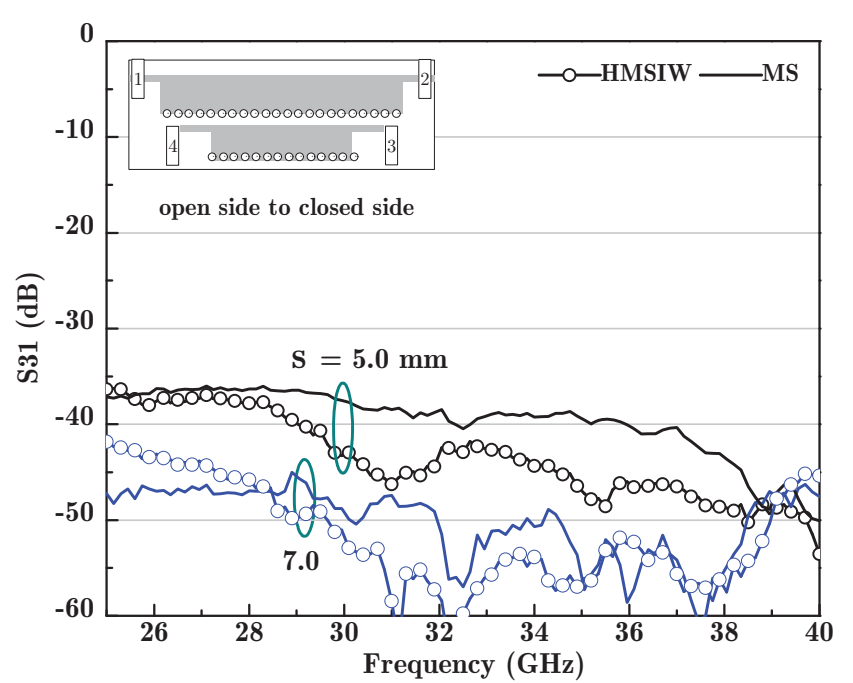

(a)

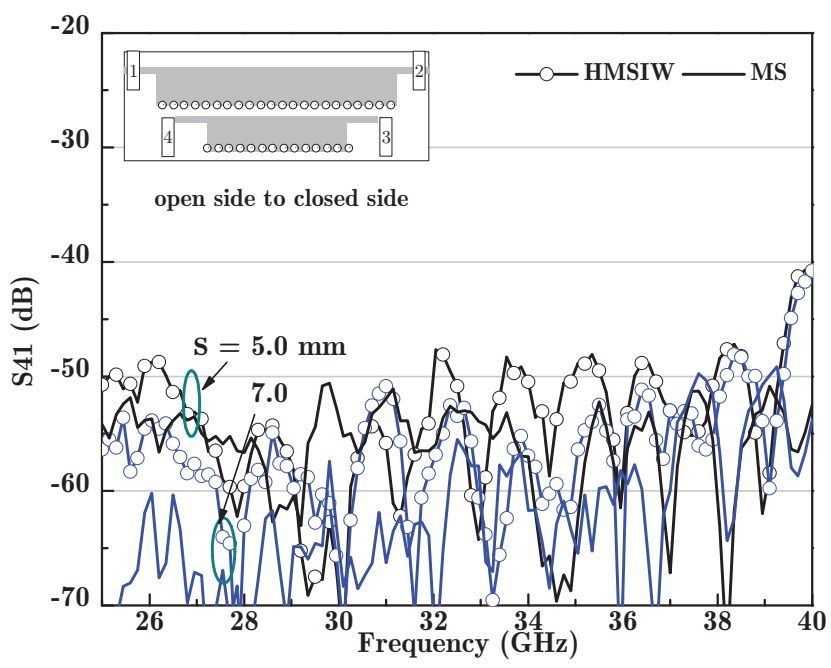

(b)

Fig. 5 Comparison of measured mutual coupling between a pair of parallel HMSIWs and a pair of parallel microstrip lines, at different interspacings of $5.0 \mathrm{~mm}$ and $7.0 \mathrm{~mm}$. The coupled HMSIWs are placed in the configuration of "open side to closed side". (a) Far-end coupling, (b) near-end coupling.

The "common closed side" configuration is the second investigated case. The spacing $s$ is fixed and equal to twice the HMSIW width $w$, i.e., $s=2 w=7.0 \mathrm{~mm}$. Fig. 4(a) and (b) present the measured coupling coefficients S31 and S41. With appropriate selection of the metallic via diameter and spacing (here $d=0.4 \mathrm{~mm}$ and $s_{v}=0.8 \mathrm{~mm}$ ), the coupled HMSIWs demonstrate a similar mutual coupling level as the coupled microstrip lines, in terms of both far-end and near-end crosstalk.

Finally, the results for the "open side to closed side" configuration are shown in Fig. 5 for the distances of $s=5.0 \mathrm{~mm}$ and $s=7.0 \mathrm{~mm}$. It is observed that, below $28 \mathrm{GHz}$, the HMSIWs produces a S31 (Fig. 5(a)) close to that of the microstrip lines for the spacing $s=5.0 \mathrm{~mm}$ and slightly higher than the microstrip for $\mathrm{s}=7.0 \mathrm{~mm}$. This can be explained by 
the power leakage from the HMSIW open edge. With increasing frequency, this power leakage decreases which results in a lower coupling coefficient S31 being measured above $28.0 \mathrm{GHz}$ for the HMSIWs, as compared to the microstrip lines. In contrast, the near-end coupling coefficient S41 shown in Fig. 5(b) does not show a clear frequency dependence, with coupling levels for both line types generally at similar levels.

The experimental results show that the pair of coupled HMSIWs in the "open side to open side" configuration encounters higher far-end coupling than the coupled microstrip lines at lower frequencies. To improve the isolation between the parallel HMSIWs, a row of metallic vias can be inserted as a "fence" in between the open edges. For the coupled HMSIWs in the structure of "common closed side", the coupling level is generally the same as that of microstrip lines. Increasing the ratio $d / s_{v}$ of metallic vias diameter to their spacing, can help suppress the mutual coupling. The third geometry of "open side to close side" exhibits the best isolation among the three configurations, and can even surpass that of coupled microstrip lines. Finally, whatever configuration is adopted, it is important to design the HMSIW with sufficient width to avoid operating the HMSIW near its cutoff frequency

\section{CONCLUSION}

This paper has experimentally investigated the mutual coupling levels between pairs of parallel HMSIWs in three possible configurations, and compared the obtained results to the crosstalk between microstrip lines. The presented results were obtained at $\mathrm{Ka}$ band, but a corresponding set of measurements at $\mathrm{X}$ band demonstrated similar behavior.
Those results should be taken into account when selecting an appropriate layout of multiple HMSIWs in the design of microwave integrated circuits (MICs) and array antenna feeds.

\section{ACKNOWLEDGMENT}

This work was partly supported by the NSFC under grant 60621002 from China. Q.H. Lai was with the Laboratory for Electromagnetic Fields and Microwave Electronics (IFH), ETH Zürich, Switzerland on leave from the State Key Lab. of Millimeter Waves (SKLMM) when part of this work was done. The authors thank Prof. R. Vahldieck, H. Benedickter M. Lanz, and C. Maccio at IFH. The authors also acknowledge Associate Prof. Zhenqi Kuai and Tianyi Huo at SKLMM for their assistance in the measurement. C. Fumeaux acknowledges the support of the Australian Research Council (ARC) Future Fellowship scheme (FT100100585).

\section{REFERENCES}

[1] Q. H. Lai, C. Fumeaux, W. Hong, and R. Vahldieck, "Characterization of the propagation properties of the half-mode substrate integrated waveguide," IEEE Trans. Microw. Theory Tech., vol. 57, no. 8, pp. 1996-2004, Aug. 2009.

[2] W. Ma, K. Wu, W. Hong, and Y. J. Cheng, "Investigations on Half mode substrate integrated waveguide for high-speed interconnect application," in Proc. IEEE MTT-S Int. Microw. Workshop Series on Art of Miniaturizing RF and Microw. Passi. Comp., Chengdu, China, Dec. 2008, pp. 120-123.

[3] Q. H. Lai, C. Fumeaux, and W. Hong, "On the transition from radiation to guiding behavior of the half-mode substrate integrated waveguide," in Proc. Asia Pacific Microw. Conf., Yokohama, Japan, Dec., 2010, pp. 1601-1604. 